\title{
Nucleic Acid Hybridization
}

National Cancer Institute

\section{Source}

National Cancer Institute. Nucleic Acid Hybridization. NCI Thesaurus. Code C16924.

Laboratory procedure in which single stranded nucleic acids are allowed to interact so

that complexes, or hybrids, are formed by molecules with sufficiently similar,

complementary sequences. By this means the degree of sequence identity can be assessed and specific sequences detected. (From Biology-online) 